\title{
Left Ventricular Non-Compaction Cardiomyopathy, Ventricular Septal Defect and Pre-excitation: A Rare Coexistence of Three Abnormities in an Adult Patient.
}

\author{
Tufan Çınar, Vedat Çiçek, Sahhan Kılıç, Emre Yalçınkaya, Murat Selçuk, Ahmet Lütfullah Orhan.
}

Health Sciences University, Sultan II. Abdülhamid Han Training and Research Hospital, Department of Cardiology, Istanbul, Turkey.

\section{Corresponding Author:}

Tufan ÇINAR, MD

Health Sciences University, Sultan II. Abdülhamid Han Training and Research Hospital, Department of Cardiology,

Tibbiye Street, Uskudar, Istanbul, Turkey.

E-mail: drtufancinar@gmail.com

ORCID ID NO: https://orcid.org/0000-0001-8188-5020

Tel: $+90-216-542-2020$

FAX: $+90-216-542-2010$

Submitted date: $6^{\text {th }}$ December 2020

Accepted date: $8^{\text {th }}$ February 2021

Cite this article as: Çınar T, Çiçek V, Kılıç S, et al. Left Ventricular Non-Compaction Cardiomyopathy, Ventricular Septal Defect and Pre-excitation: A Rare Coexistence of Three Abnormities in an Adult Patient. Nepalese Heart Journal 2021; Vol 18 (1): 57-59.

\section{Abstract}

\begin{abstract}
A congenital cardiac condition characterized by ventricular trabeculations and intertrabecular recesses is described as a non-compaction cardiomyopathy. In clinical practice, since it can be associated with severe mortality and morbidity due to progressive heart failure, thromboembolic events, and fatal arrhythmias, it is important to consider non-compaction cardiomyopathy. In this case, we have an adult patient who underwent surgery due to a ventricular septal defect (VSD) and who was later diagnosed with left ventricular non-compaction cardiomyopathy (LVNC) and pre-excitation. To our knowledge, this is the first case of VSD, LVNC, and pre-excitation simultaneously present in an adult patient.
\end{abstract}

Keywords: Non-Compaction Cardiomyopathy, Pre-Excitation,VSD.

DOI: https://orcid.org/10.3126/njh.v18i1.36787

\section{Introduction}

Non-compaction cardiomyopathy is a rare congenital cardiac disease characterized by ventricular trabeculations and intertrabecular recesses. The underlying pathology is the failure of normal embryonic development of the myocardium from loosely arranged muscle fibers to mature compressed myocardium. In clinical practice, it is important to recognize a non-compaction cardiomyopathy because it can be associated with significant mortality and morbidity due to progressive heart failure, thromboembolic events, and fatal arrhythmias. In this case, we present an adult patient who was operated due to ventricular septal defect (VSD), and later diagnosed with left ventricular non-compaction cardiomyopathy (LVNC) and pre-excitation. To our knowledge, this is the first case of VSD, LVNC and pre-excitation simultaneously present in an adult patient.

\section{Case Presentation}

A 28-year-old male patient who had been previously operated for VSD presented to the cardiology outpatient clinic with a complaint of a typical chest pain. The patient did not have any other significant medical history. On physical examination, a 2/6 systolic murmur was heard on the apical region, and pre-excitation was observed on his electrocardiography (ECG) (Figure1). Transthoracic echocardiography (TTE) was performed, revealing a normal left ventricle (LV) systolic ejection fraction, mild mitral regurgitation, increased echogenicity in the interventricular septum that was compatible with a previous VSD operation, and increased trabeculations on the apical region of the LV (Figure 2). On cardiac magnetic resonance imaging (MRI), there were increased trabeculations in the LV apical and lateral regions on short-axis and long-axis view (Figure 3 and 4). The patient was diagnosed with LVNC following the cardiac MRI study. The patient underwent a cardiovascular exercise test

(a) Nepalese Heart Journal. Nepalese Heart Journal retain copyright and works is simultaneously licensed under Creative Commons Attribution License CC - By 4.0 that allows others to share the work with an acknowledge of the work's authorship and initial publication in this journal 
with the Bruce protocol, which demonstrated the disappearance of pre-excitation waves during the peak exercise stage. 24-hours Holter monitoring did not reveal any significant arrhythmias. Since the pre-excitation and LVNC carry high risk of sudden death, electrophysiological study, including ablation, was offered. However, the patient declined the procedure against medical advice.

Figure 1: Electrocardiograph of the patient. Note the presence of Delta Waves (Arrows).

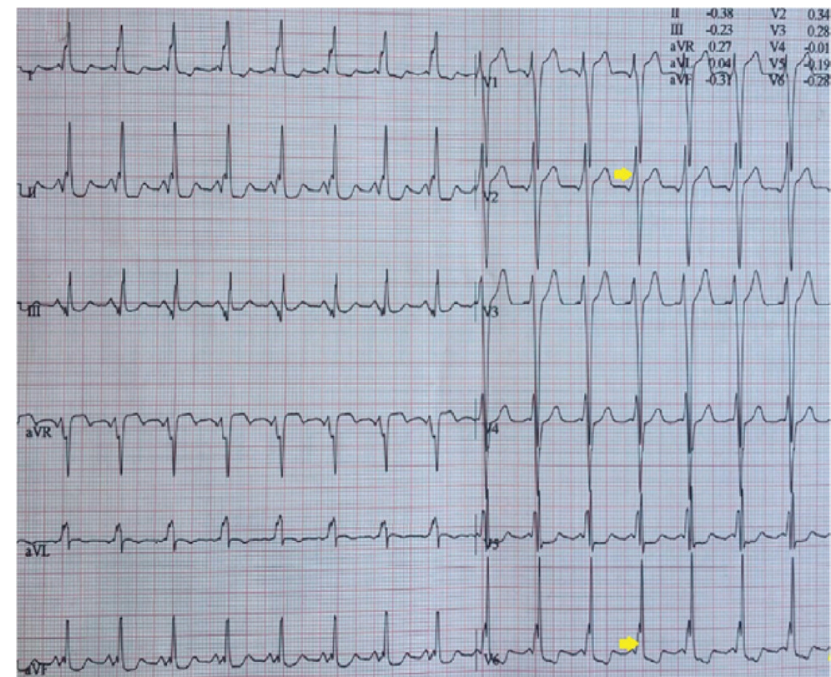

Figure 2: Transthoracic Echocardiograph Image demonstrating increased trabeculations on the Apical Region of the Left Ventricle (Arrow)

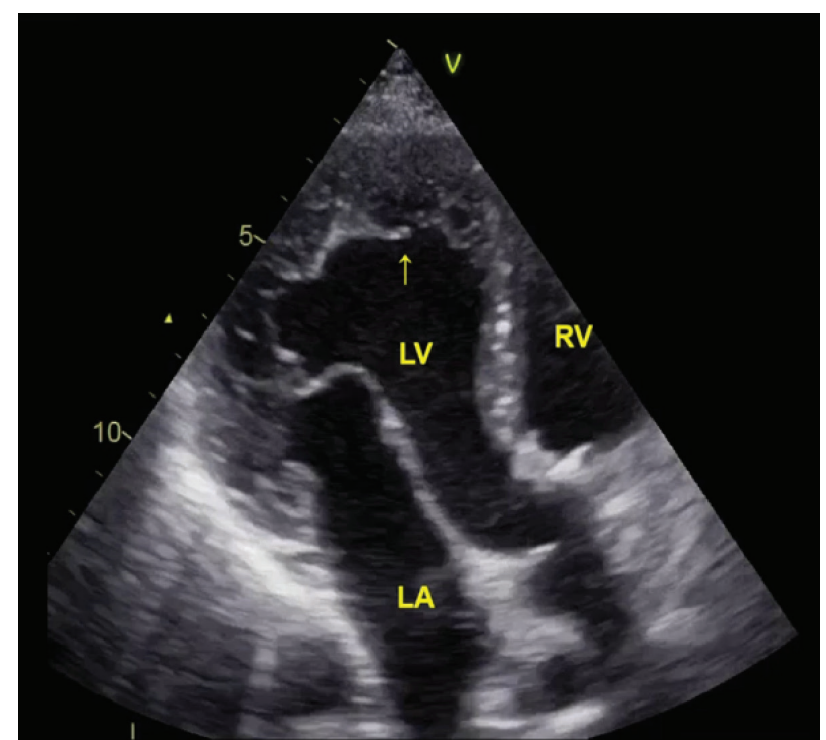

Figure 3 and 4: Short-Axis and Long-Axis view of Cardiac Magnetic Resonance Image demonstrating increased trabeculations on the Apical Region of the Left Ventricle (Arrows).
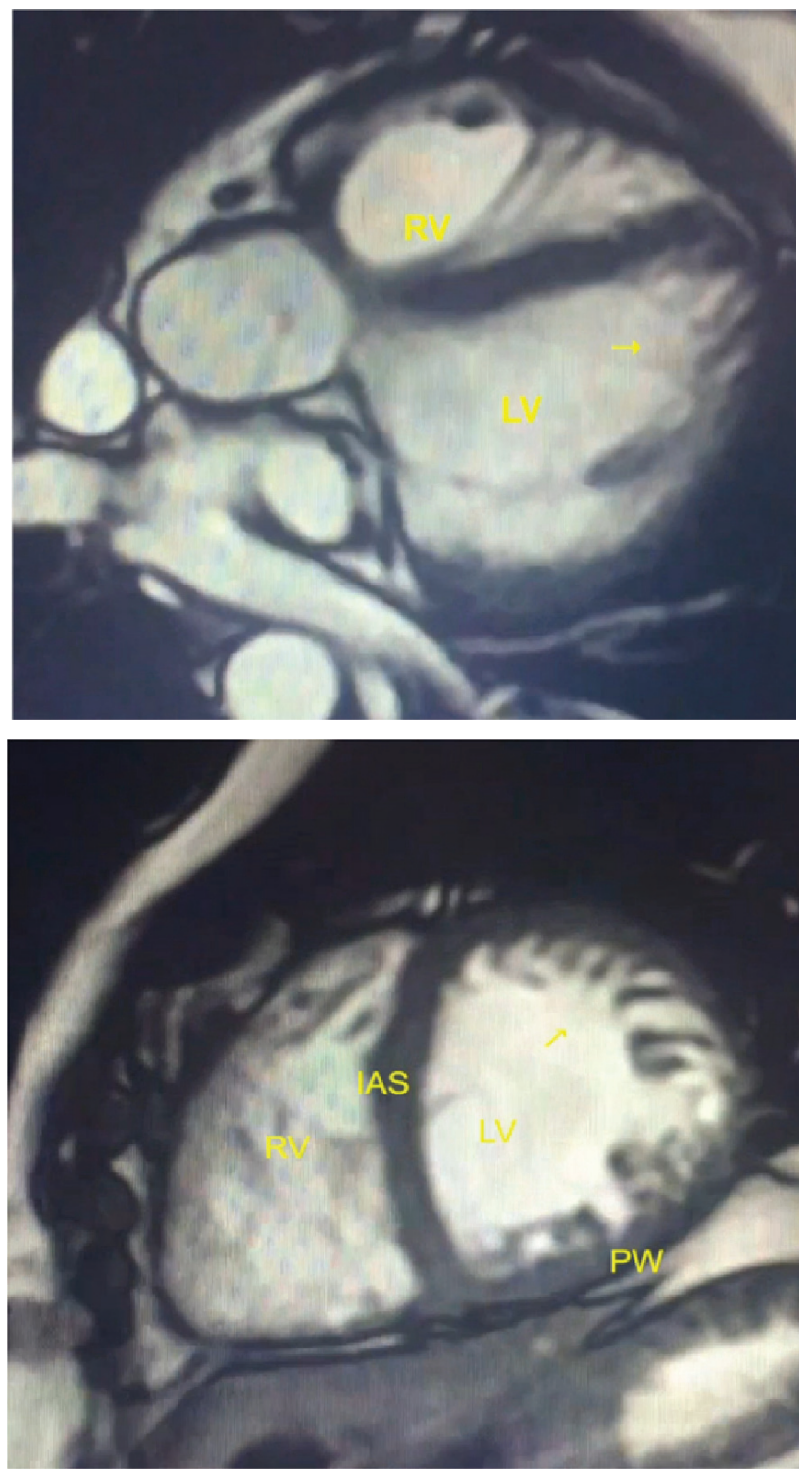

\section{Discussion}

The clinical manifestations of LVNC vary widely from asymptomatic phase to progressive congestive heart failure, fatal arrhythmias, and systemic thromboembolism. Although the prevalence of LVNC in the general population is not known exactly, in a study conducted in a Switzerland, it was reported that its prevalence was $0.014 \% .{ }^{1}$ LVNC can sometimes accompany other congenital heart defects, such as bicuspid aortic valve, Ebstein's 
anomaly, congenitally corrected transposition of great arteries, hypoplastic left heart syndrome, and left atrial appendix isomerism, and with VSD, as it was shown in our patient. ${ }^{2,3}$ The most common findings in patients with LVNC are non-specific abnormalities of the resting ECG. It was reported that $94 \%$ of adult patients might have abnormal ECG findings due to progressive endomyocardial fibrosis. ${ }^{1}$ Similarly, in our case, the patient had a pre-excitation abnormality.

In the present case, we report an adult patient with LVNC, operated with VSD, and pre-excitation, which has not been demonstrated until now. Interestingly, the diagnosis of LVNC had been missed until our evaluation. Hence, this case emphasizes that cardiologists should be aware of the presence of LVCN in patients with congenital cardiac defects, including VSD.

\section{Acknowledgment : None}

Conflict of Interest: There is no conflict of interest to declare.

\section{References}

1. Swinkels BM, Boersma LV, Rensing BJ, et al. Isolated left ventricular noncompaction in a patient presenting with a subacute myocardial infarction. Neth Heart J. 2007; 15:10911. https://doi.org/10.1007/BF03085964

2. Jenni R, Oechslin E, Schneider J, et al. Echocardiographic and pathoanatomical characteristics of isolated left ventricular non-compaction: a step towards classification as a distinct cardiomyopathy. Heart. 2001; 86:666-71. https://doi.org/10.1136/heart.86.6.666

3. Elliott $\mathrm{P}$, Andersson B, Arbustini E, et al. Classification of the cardiomyopathies: a position statement from the European Society of Cardiology Working Group on Myocardial and Pericardial Diseases. Eur Heart J. 2008;29:270-6. https://doi.org/10.1093/eurheartj/ehm342 